\title{
XXX. Verbindung des Spektralapparates mit dem Axenwinkelapparate.
}

\author{
Von
}

Victor $\nabla$. Lang in Wien.

Ich habe in einen vor Kurzem erschienenen Aufsatze ${ }^{*}$ gezeigt, dass die Dispersion der optischen Elasticitätsaxen des Gypses in der Symmetriebene anormal ist, und dass gleichzeitig der Winkel der optischen Axen für die Linie $D$ ein Maximum hat. Von beiden überzeugt man sich durch Untersuchung einer primären Axenplatte ${ }^{* *}$ ) nach der von G. Kirch hoff***) am Aragonite angewandten Methode. Nur wird man hierbei zur Erzeugung des Spektrums jetzt einen Spektralapparat verwenden, die sich ja seither so verbreitet haben. Auch kann man stalt des von Kirchhoff angewandten Theodoliten zur Messung des Axenwinkels gleich einen vollständigen Axenwinkelapparat benutzen. Das Beleuchtungsrohr dieses Apparates muss aber hierbei vor den Spektralapparat gesetzt werden, weil die Gypsplatte nahezu im zweiten Brennpunkt des Oculars des Spektralapparats stehen muss, soll Spektrum und Axenbild gleichzeitig gut zu sehen sein. Hierzu muss natürlich noch das Fadenkreuz des Axenwinkelapparates mit dem des Spektralapparates coincidiren.

Durch Drehen des Prisma des Spektralapparates kann man dann eine beliebige Fraunhofer'sche Linie zur Coïncidenz mit dem letzteren Fadenkreuz bringen und hierauf den Axenwinkel für diese Linie bestimmen.

Beobachtet man eine der Axen des Gypses, während man durch Drehen des Prisma die verschiedenen Theile des Spektrums ins Gesichtsfeld bringt,

*) Sitzungsber. d. Wiener Akad. 76. (Ausz. in diesem Heft, XXXI 2, S. 499-501.)

**) d. i. eine zur erster Mittellinie senkrechte Platte: secundäre Axenplatte wäre eine zur zweiten Mittellinie senkrechte Platte. Ebenso kann man zwischen positiver und negativer Axenplatte unterscheiden.

***) Poggend. Annalen. 10S, S. 567. 
so sieht man, dass die Lage des Axenbìldes gegen das Fadenkreuz sich nicht immer in demselben Sinne ändert, sondern dass die mmatte Axe bei der Linie $D$, die mrolhe aber zwischen $E$ und $F$ einen Umkehrpunkt hat. Namentlich Ersteres ist sehr gut wahrzunehmen und es wird hierdurch der allfällige Vorwurf widerlegt, dass jenes anormale Verhalten des Axenwinkels eine Folge von Temperaturschwankungen bei den einzelnen Messungen wäre.

Das Drehen des Prisma bei letzterer Beobachtung bietet aber, da es bei den gewöhnlichen Spektralapparaten aus freier Hand zu geschehen hat, einige Schwierigkeit, und ich habe daher zur Vermeidung desselben die in beistehender Figur gezeichnete Verbindung des Spektralapparates mit dem Axenwinkelapparat ausgeführt, wodurch letzterer gleichzeilig mil dem Beobachtungsfernrohr des Spektralapparates bewegt wird, und das Prisma auf dem Minimum der Deviation stehen bleibt.

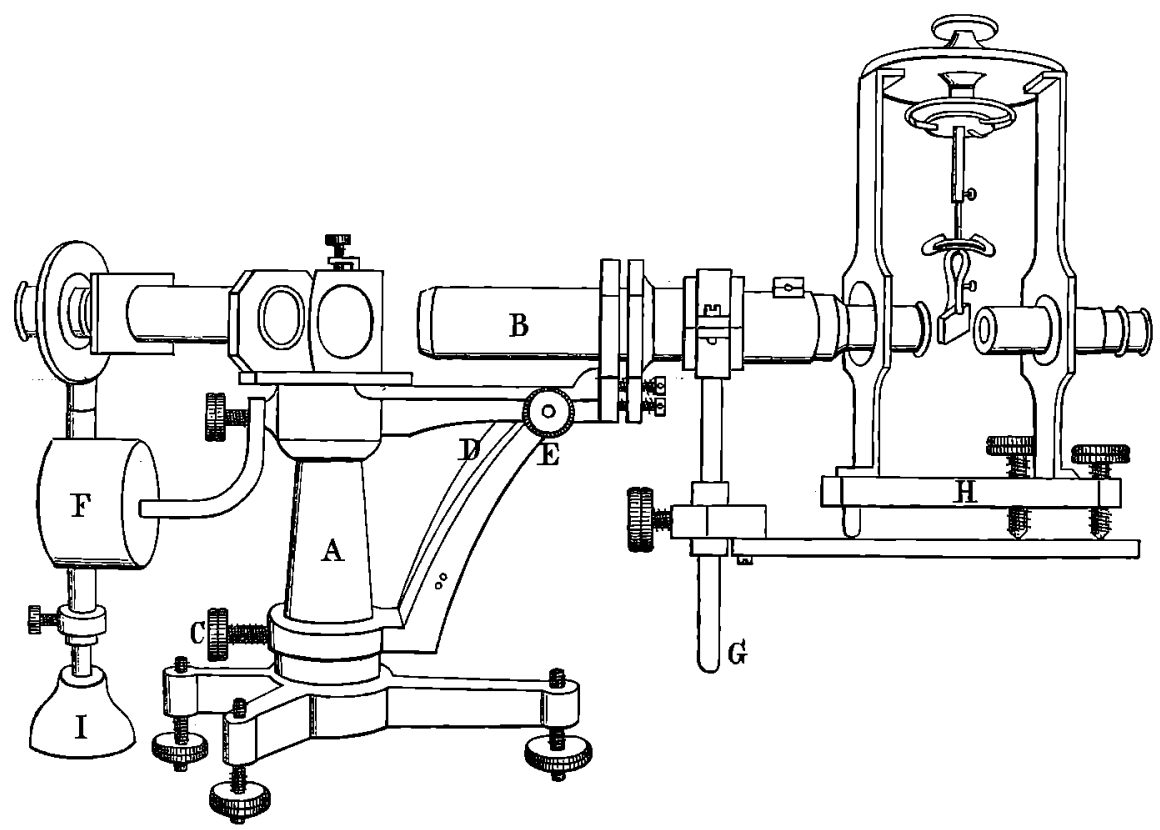

Zur Erläuterung der Zeichnung ist wohl nur wenig beizufügen. Der Axenwinkelapparat $H$ steht auf einem Träger $G$, welcher an das Beobachtungsfernrohr $B$ des Spektralapparates $A$ mit zwei Schrauben befestigt wird. Aehnliche Träger benutzı man auch, um vor das Spaltrohŕ absorbirende Körper etc. zu stellen. Die Bewegung des Fernrohrs geschieht am Zweckmässigsten, indem man den Träger an seinem untern Ende bei $G$ anfasst. 
Das Gewicht des Axenwinkelapparales wird durch das Gegengewicht $F$ balancirt, und das Beleuchtungsrohr desselben, bestehend aus Nicol und Linse, sammt seiner Hülse auf dem separaten Statif $I$ vor die Spalte gestellt.

Will man den combinirten Apparat nicht nur zu den fruher erwähnten qualitativen Versuchen benutzen, sondern um wirklich den Axenwinkel einer Platte für verschiedene Fraunhofer'sche Linien zu messen, so muss wohl derSpektralapparat mit einer feinen Einstellung versehen sein, womit das Fadenkreuz des Fernrohrs $B$ genau auf eine beliebige Fraunhofer'sche Linie eingestellt werden kann. Zu dem Zwecke liess ich an meinem A pparate ein drehbares Messingstück anbringen; welches durch die Schraube $C$ festgeklemmt werden kann. Dasselbe trägt die Mikrometerschraube $E$, wodurch das Fernrohr sammt Axenwinkelapparat fein bewegt wird, indem eine Feder $D$ das Fernrohr gegen die Schraube $E$ druckt.

Was den Axenwinkelapparat betrifft, so habe ich dessen Beschreibung Sitzber. d. Wiener Akad. Bd. 55 und Carl's Repert. Bd. 3 gegeben. 